\title{
A Priori Laplacian with Hamming Distance: Advanced Dimension Reduction Technique
}

\author{
Gaurav Gupta \\ Department of Electronics and Communication Engineering \\ Maharishi Markandeshwar University (M.M.U) \\ Sadopur (Ambala)-134007, India
}

\begin{abstract}
Images containing faces are essential to intelligent visionbased human computer interaction, and research efforts in face processing include face recognition, face tracking, pose estimation, and expression recognition. The rapidly expanding research in face processing is based on the premise that information about a user's identity, state, and intent can be extracted from images and that computers can then react accordingly, e.g., by knowing person's identity, person may be authenticated to utilize a particular service or not. A first step of any face processing system is registering the locations in images where faces are present. However, face registration for whole database is a challenging task because of variability in scale, location, orientation (up-right, rotated), and pose (frontal, profile). Facial expression, occlusion, and lighting conditions also change the overall appearance of face. The Image registration algorithm will register all these images present in the database. The face recognition algorithm which is insensitive to large variation in lighting direction and facial expression is to be implemented. Taking a pattern classification approach, each pixel in an image can be considered as a coordinate in a high-dimensional space. The advantage of this is that the images of a particular face, under varying illumination but fixed pose, lie in a 3D linear subspace of the high dimensional image space-if the face is a Lambertian surface. However, since faces are not truly Lambertian surfaces and do indeed produce self-shadowing images will deviate from this linear subspace. Rather than explicitly modeling this deviation, project the image into a subspace in such a manner which discounts those regions of the face with large deviation. This is achieved by using dimension reduction techniques like Principal component analysis (PCA), Linear Discriminant analysis (LDA), Laplacian faces and other modified approaches like A Priori Laplacian and A Priori Laplacian with Hamming Distance.
\end{abstract}

\section{General Terms}

Face Recognition, Image Processing, Pattern Recognition

\section{Keywords}

Hamming Distance, Principal component analysis, Linear discriminant analysis, Linear projective projection, Eigen value, Laplacian faces, A Priori Laplacian

\section{INTRODUCTION}

Face recognition has been an active research area over the last 30 years. It has been studied by scientists from different areas of psychophysical sciences and those from different areas of computer sciences. Psychologists and neuroscientists mainly deal with the human perception part of the topic, whereas engineers studying on machine recognition of human faces deal with the computational aspects of face recognition. Face recognition has applications mainly in the fields of biometrics, law enforcement, access Control and security and surveillance systems.

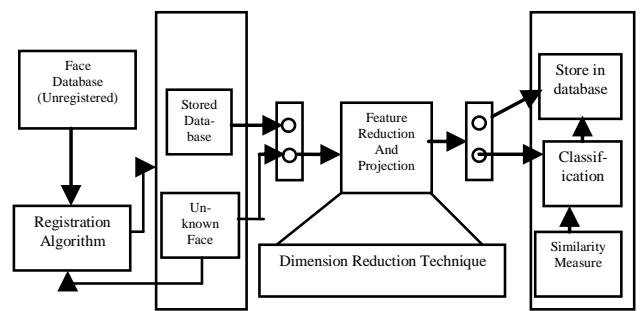

Figure1 Block Diagram of Face Recognition System

The face is our primary focus of attention in social intercourse, playing a major role in conveying identity and emotion. Although the ability to infer intelligence or character from facial appearance is suspect, the human ability to recognize faces is remarkable. We can recognize thousands of faces learned throughout our lifetime and identify familiar faces at a glance even after years of separation. This skill is quite robust, despite large changes in the visual stimulus due to viewing conditions, expression, aging, and distractions such as glasses, beards or changes in hair style. Face recognition has become an important issue in many applications such as security systems, credit card verification and criminal identification. For example, the ability to model a particular face and distinguish it from a large number of stored face models would make it possible to vastly improve criminal identification. Although it is clear that people are good at face recognition, it is not at all obvious how faces are encoded or decoded by the human brain. Unfortunately developing a computational model of face recognition is quite difficult, because faces are complex, multi-dimensional visual stimuli. Therefore, face recognition is a very high level computer vision task, in which many early vision techniques can be involved.

The first step of human face identification is to extract the relevant features from facial images. Research in this field primarily intends to generate sufficiently reasonable familiarities of human faces so that another human can correctly identify the face. The question naturally arises as to how well facial features can be quantized. If such a quantization if possible then a computer should be capable of recognizing a face given a set of features. Investigations by numerous researchers over the past several years have indicated that certain facial characteristics are used by human beings to identify faces.

The block diagram of system is shown in Figure 1 which explains about the step by step procedure for Training and Testing of face images. The initial step is the Registration of images present in database. Once all images are registered 
they are applied to dimension reduction block where the most important dimension are kept and then for classification it goes to classification block where different similarity measures are used to classify the test image.

\section{MOTIVATION}

Today security and surveillance systems are of major importance in high risk areas like military, companies etc. In surveillance system face recognition is an important step for better and accurate surveillance. The complexity involves in it are high dimension subspace, variation of expressions, lighting, size etc motivates to develop a new and better algorithm which really enhance the security of such systems. The necessity for personal identification in the fields of private and secure systems made face recognition one of the main fields among other biometric technologies. The importance of face recognition rises from the fact that a face recognition system does not require the cooperation of the individual while the other systems need such cooperation. Face recognition algorithms try to solve the problem of both verification and identification. When verification is on demand, the face recognition system is given a face image and it is given a claimed identity. The system is expected to either reject or accept the claim. On the other hand, in the identification problem, the system is trained by some images of known individuals and given a test image. It decides which individual the test image belongs to. The main problem of face recognition is its high dimension space which is to be reduced by any dimension reduction techniques. The pattern recognition [8] approach then tries to match the face features which are extracted from all the images present in the database. So there are two major problems one is feature extraction and then pattern recognition. Before this image registration of all the faces is required to enhance the recognition rate of whole system. So these all motivates to search for new method to solve all these problems and then integrate them to make functional system with high accuracy.

\section{SCOPE OF THE PAPER}

In this paper, Laplacian faces which uses linear projective projection (LPP) is studied and finally enhanced before accuracy. LPP is designed for preserving local structure; it is likely that a nearest neighbour search in the low dimensional space will yield similar results to that in the high dimensional space. LPP's are linear projective maps that arise by solving a variational problem that optimally preserves the neighborhood structure of the data set. Finally the algorithm is modified to yield better results in terms of time and accuracy.

\section{LAPLACIAN FACES}

An appearance-based face recognition method called the Laplacian face [5] [6] approach using Locality Preserving Projections (LPP), the face images are mapped into a face subspace for analysis. Different from Principal Component Analysis (PCA) and Linear Discriminant Analysis (LDA) [2] [8] which effectively see only the Euclidean structure of face space, LPP finds an embedding that preserves local information, and obtains a face subspace that best detects the essential face manifold structure. The Laplacian faces are the optimal linear approximations to the Eigen functions [3] of the Laplace Beltrami operator on the face manifold. In this way, the unwanted variations resulting from changes in lighting, facial expression, and pose may be eliminated or reduced.

\section{LOCALITY PRESERVING PROJECTIONS (LPP)}

LPP finds an embedding that preserves local information, and obtains a face subspace that best detects the essential face manifold structure. The concept of Locality Preserving Projections (LPP) [4] for face recognition is new one. If one wishes to retrieve audio, video, text documents under a vector space model, then one will ultimately need to do a nearest neighbour search in the low dimensional space. Since LPP is designed for preserving local structure, it is likely that a nearest neighbour search in the low dimensional space will yield similar results to that in the high dimensional space. LPP's are linear projective maps that arise by solving a variational problem that optimally preserves the structure of the data set.

Generally used dimension reduction techniques like PCA and LDA [2] aim to preserve the global structure. However, in Identity retrieval applications, the local structure is more important. So Locality Preserving Projection (LPP) is used for learning a locality preserving subspace. LPP seeks to preserve the intrinsic geometry of the data and local structure. The objective function of LPP is as follows:

$$
\min \sum\left(\mathrm{y}_{\mathrm{i}}-\mathrm{y}_{\mathrm{j}}\right)^{2} \mathrm{~S}(\mathrm{i}, \mathrm{j})
$$

Where $y_{i}$ and $y_{j}$ are the image $i$ and $j$ in reduced Dimension space and $S$ is the weight matrix in which weight $S(i, j)$ are according to similarity between $\mathrm{i}^{\text {th }}$ and $\mathrm{j}^{\text {th }}$ image in original dimension space.

The minimization of objective function can be achieved by posing this problem as Eigen Value problem.

$$
\begin{aligned}
\mathrm{y}_{\mathrm{i}} & =\mathrm{W}^{\mathrm{T}} \mathrm{X}_{\mathrm{i}} \\
F & =\frac{1}{2} \sum_{i j}\left(y_{i}-y_{j}\right)^{2} S(i, j) \\
F & =\frac{1}{2} \sum_{i j}\left(W^{T} X_{i}-W^{T} X_{j}\right)^{2} S(i, j) \\
F & =\sum_{i j} W^{T} X_{i} S(i, j) X_{i}^{T} W-\sum_{i j} W^{T} X_{i} S(i, j) X_{j}^{T} W \\
F & =\sum_{i} W^{T} X_{i} D(i, i) X_{i}^{T} W-W^{T} X S X^{T} W \\
\mathrm{~F} & =\mathrm{W}^{\mathrm{T}} X D X^{T} W-W^{T} X S X^{T} W \\
\mathrm{~F} & =\mathrm{W}^{\mathrm{T}} \mathrm{XLX}^{\mathrm{T}} \mathrm{W}
\end{aligned}
$$

where $D(i, i)=\sum_{j} S(i, j)$ ie sum of rows(columns)and L=D-S The larger the value of $\mathrm{D}(\mathrm{i}, \mathrm{i})$ (corresponding to $\mathrm{y}_{\mathrm{i}}$ ) is, the more "important" is $\mathrm{y}_{\mathrm{i}}$. The Transformation vector $\mathrm{W}$ that minimizes the objective function is given by the minimum eigen value [3] solution to the generalized eigen value problem: Finally, the minimization problem reduces to finding

$$
\arg \min _{\mathrm{W}} \mathrm{XLX}^{\mathrm{T}} \mathrm{W}
$$

Under constraint $\mathrm{XDX}^{\mathrm{T}} \mathrm{W}=1$

$$
\mathrm{XLX}^{\mathrm{T}} \mathrm{W}=\lambda \mathrm{XDX}^{\mathrm{T}} \mathrm{W}
$$

The solution of this equation will give the optimum value of $\mathrm{W}$ which will minimize the objective function. This $\mathrm{W}$ will be the transform vector from $\mathrm{N}^{2}$ Dimension to lower dimension. The basis images (vectors) are called LAPLACIAN faces as they are derived from Laplace Beltrami operator. 


\subsection{ALGORITHM}

Apply the PCA to all images in database so as to reduce the dimension from $\mathrm{N}^{2}$ to lower dimension $\mathrm{M}\left(\mathrm{M}<<\mathrm{N}^{2}\right)$.

A. Create a Binary graph in which value will be ' 1 ' for $\mathrm{K}$ nearest Neighbors and ' 0 ' for others. In $K$ nearest approach Graph is generated using $\mathrm{K}$ nearest Neighbor in original Domain.

B. Assign weights to the branches which are connected to each other. The weight matrix $\mathrm{S}$ can be of various types which are discussed in next section.

C. From Weight matrix S calculate D and L matrix.

D. Solve the equation (1) for $\mathrm{W}$ and Select lowest k no. of Eigen Vectors [7]

E. Transpose whole Database into lower $\mathrm{k}$ dimension subspace.

\subsection{FACE RECOGNITION USING LAPLACIAN FACES}

Whenever a new face comes:

A. Transpose it with same $\mathrm{W}$ vector.

B. Apply Nearest Neighbour Pattern classification [9] approach to retrieve identity of the person from database.

\subsection{SIMILARITY MATRIX}

\section{A. Binary face}

In this Similarity matrix is Binary one with weights equal to "One" if it is nearest neighbour or a class member otherwise "Zero".

$$
\begin{aligned}
\mathrm{S}(\mathrm{i}, \mathrm{j}) & =1 \text { if Nearest neighbour } \\
& =0 \text { otherwise }
\end{aligned}
$$

\section{B. Euclidean}

In this case weight will be more if they are close in Euclidean Space ie inversely proportional to Euclidean Distance. It lies between 0 and 1 .

$$
\begin{aligned}
& S(i, j)=\exp \left(-\left(X_{i}-X_{j}\right)^{2} / t\right) \text { if Nearest neighbor } \\
& =0 \quad \text { otherwise }
\end{aligned}
$$

where $t$ is suitable constant.

\subsection{RESULTS}
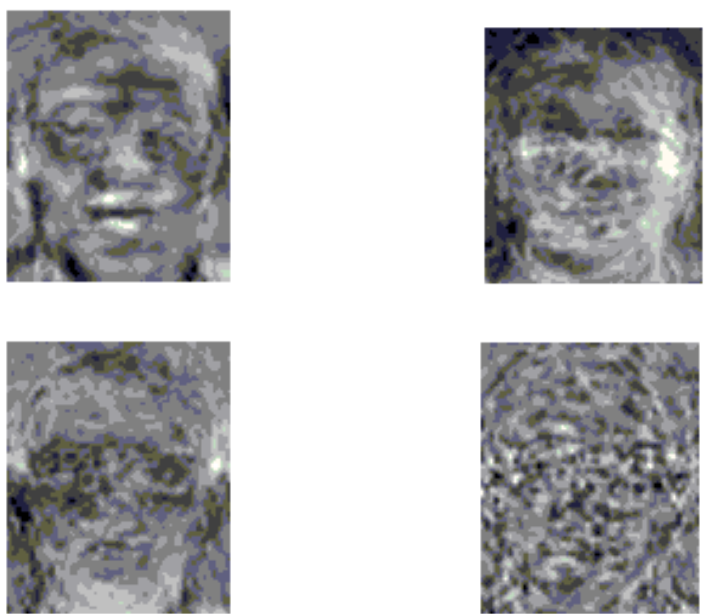

Figure2. Top Four Laplacian Faces

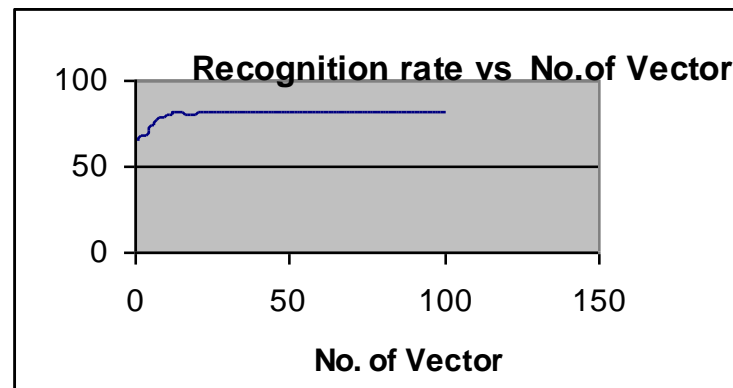

Figure 3 Recognition rate Vs No. of Vector 6. A PRIORI LAPLACIAN CONCEPT

Locality Preserving Projections (LPP) finds an embedding that preserves local information, and obtains a face subspace that best detects the essential face manifold structure. Since LPP is designed for preserving local structure, it is likely that a nearest neighbor search in the low dimensional space will yield similar results to that in the high dimensional space. LPP's are linear projective maps that arise by solving a variational problem that optimally preserves the neighborhood structure of the data set. In this approach [1] the Laplacian is used but prior knowledge of each face to its class is used, which removes the Nearest Neighbor search from the Laplacian. The removal of this step enhances its capability in terms of time. So it is a combination of Laplacian and LDA in which LPP tries to preserve the local structure and LDA tries to match the face using Mahalanobis Distance. The objective function is

$$
\begin{aligned}
& \min \sum\left(y_{i}-y_{j}\right)^{2} S(i, j) \\
& \begin{aligned}
& G(i, j)=1 \text { if face } i \text { and } j \text { are of same class } \\
&= 0 \text { otherwise } \\
& S(i, j)=\exp \left(-\left(X_{i}-X_{j}\right)^{2} / t\right) \text { if class member } \\
&=0 \quad \text { otherwise }
\end{aligned}
\end{aligned}
$$

Where $S(i, j)$ is the similarity measure between two images and $j$ and $y$ is the image in reduced dimension space. In this case 2-D Graph $G$ is a Binary Graph, which has ' 1 ' as its value if two images are class member otherwise ' 0 '. After this the similarity measure is computed for class members only. Then equation (2) is solved for Basis vectors which are further used to project the original images to reduced dimension space. If any test face comes that is reduced to the same space using same vectors. In reduced dimension space the test face is classified to one of the given class using Mahalanobis distance. 


\subsection{RESULTS}
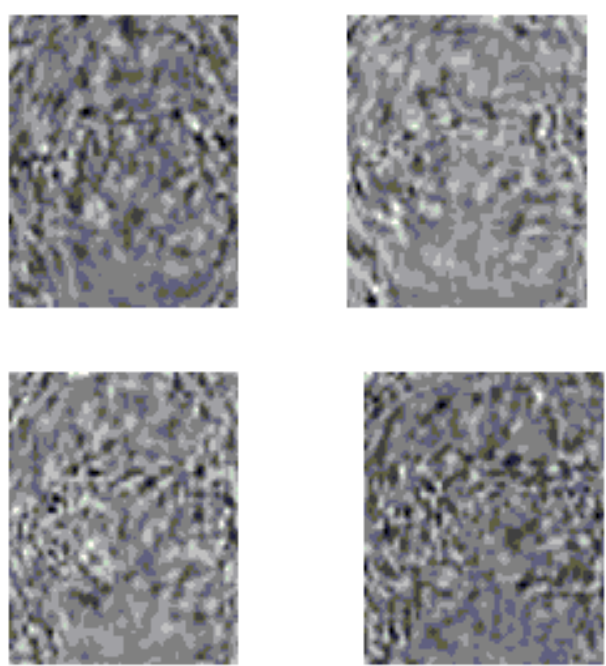

Figure 4 Top four priori Laplacian Faces

Above figure shows the best basis faces for the database from which all the faces can be reconstructed. Figure 5 shows the recognition rate with respect to number of vectors chosen for recognition. For this also after a particular number there is no improvement in the recognition rate. So again the proper number of vectors is to be chosen for better performance.

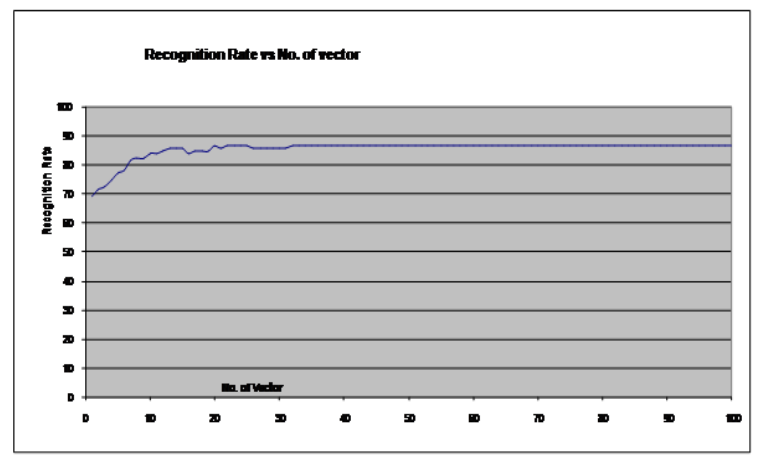

Figure 5 Recognition Rate vs No. of Vector for a priori Laplacian

\section{A PRIORI LAPLACIAN WITH HAMMING DISTANCE}

In this algorithm similarity measure which is Euclidean distance in case of Laplacian faces and a priori Laplacian is replaced by a new and better similarity measure known as hamming Distance. The problem with Binary and Euclidean similarity measures is that they are not so good similarity measure in case of Face space because of high dimensionality so a new good and efficient similarity measure is required. So the proposed similarity measure which is based upon the Hamming Distance of the $3 \times 3$ Neighbor of each pixel. In this case weights are to be calculated on the basis of Hamming Distance between $3 \times 3$ Neighborhoods of each pixel in the image. For each pixel $(i, j)$ Eight neighborhood is compared with intensity value at center. If it is greater than that it is assigned ' 1 ' else ' 0 '.

\begin{tabular}{|c|c|c|c|c|c|}
\hline $\mathrm{i}-1, \mathrm{j}-1$ & $\mathrm{i}-1, \mathrm{j}$ & $\mathrm{i}-1, \mathrm{j}+1$ & 1 & 0 & 1 \\
\hline$i, j-1$ & $\mathrm{i}, \mathrm{j}$ & $\mathrm{i}, \mathrm{j}+1$ & 1 & $X$ & 0 \\
\hline$i+1, j-1$ & $\mathrm{i}+1, \mathrm{j}$ & $i+1, j+1$ & 0 & 1 & 0 \\
\hline
\end{tabular}

$$
\begin{aligned}
D_{s, t}(i, j, p) & =1 \text { if } I(i+s, j+t)>=I(i, j) \text { for } s, t=-1,0,1 \\
& =0 \text { otherwise }
\end{aligned}
$$

This equation stores one bit corresponding to each neighbor thus whole result is one byte for a given pixel $(i, j)$ in the image $q$. In this $(0,0)$ location is skipped because that is always 1 .

$H(i, j, p, q)=$ Hamming $\operatorname{distance}(D(i, j, p), D(i, j, q))$ Hamming distance is just the No of Bit change between two Binary words. So $\mathrm{H}$ is calculated for all pairs which have value 1 in the Graph G. After calculating hamming Distance for each pixel they are added, Normalized with respect to Max. For each pixel store its neighborhood binary pattern and then for similarity between $\mathrm{p}$ and $\mathrm{q}$ face can be computed by adding Hamming Distance for each pixel in the image. This similarity measure actually inversely proportional to the similarity that is more similar ones will have less weight and vice versa.

Mathematically Similarity Matrix

$$
S(p, q)=\sum_{\mathrm{i}} \sum_{i} H(i, j, p, q)
$$

Where $H(i, j, p, q)$ is Hamming Distance of Neighborhood of pixel location $i, j$ for the images $\mathrm{p}$ and $\mathrm{q}$. Once Similarity measure $\mathrm{S}$ is computed for all images, below equation is solved using Eigen concept as explained earlier in the Block diagram.

$$
\min \sum\left(y_{i}-y_{j}\right)^{2} S(i, j)
$$

Then above equation is solved for Basis vectors which are further used to project the original images to reduced dimension space. If any test face comes that is reduced to the same space using same vectors. In reduced dimension space the test face is classified to one of the given class using Mahalanobis distance.

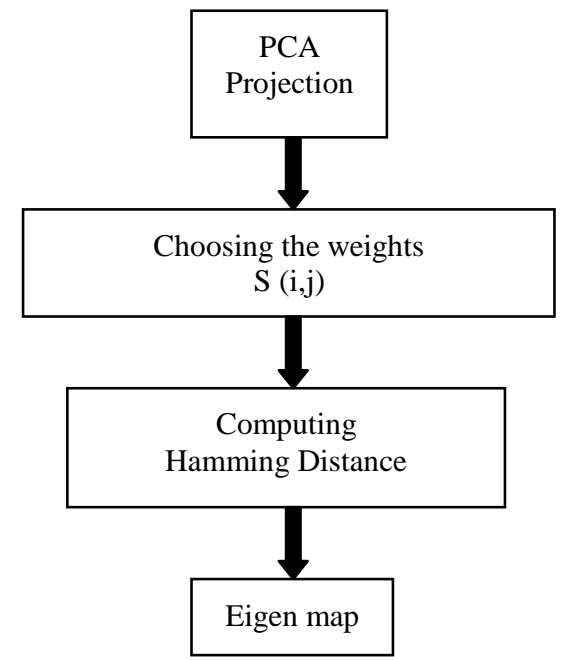

Fig 6 Flow Chart for a priori Laplacian with Hamming Distance 


\subsection{RESULTS}
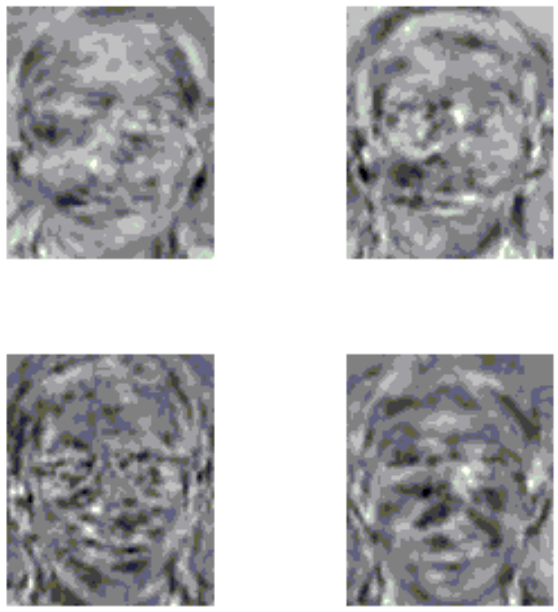

Figure 7 Top Four Basis Images for a priori Laplacian with Hamming Distance

The figure 7 shows the best basis faces for this algorithm which is used for recognition purpose. Figure 8 shows the graph between recognition rate and the number of vectors required to reduce the dimension. The recognition rate is high as compare to the previous algorithm which uses Euclidean distance as the similarity measure.

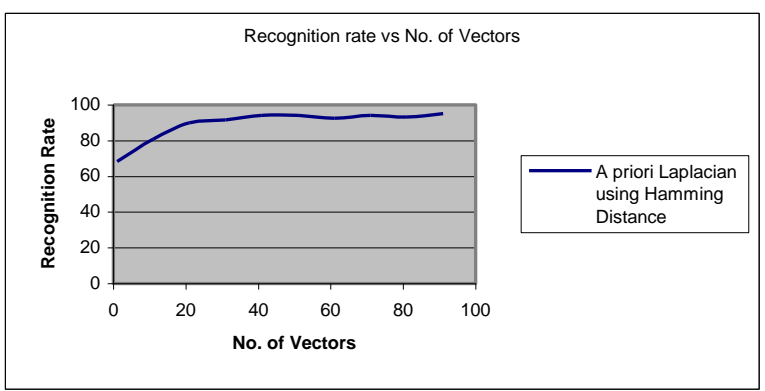

Figure 8 Recognition Rate vs No. of Vector for a priori Laplacian with hamming Distance

\section{CONCLUSION}

The manifold ways of face analysis are introduced in this paper in order to detect the underlying nonlinear manifold structure in the manner of linear subspace learning. In reference to Laplacian faces this concept which uses labeled samples, a different and effective similarity measure (Hamming Distance) works very well on the database for the purpose of recognition of identity of a person. The accuracy rate also increases due the effect of class concept i.e. labeled samples which are absent in Laplacian faces concept. Also the computational complexity is reduced due to no more searches for $\mathrm{k}$ nearest neighbor for each sample. This comparison shows that A Priori Laplacian with Hamming Distance is best in terms of recognition rate while a priori Laplacian is the next.

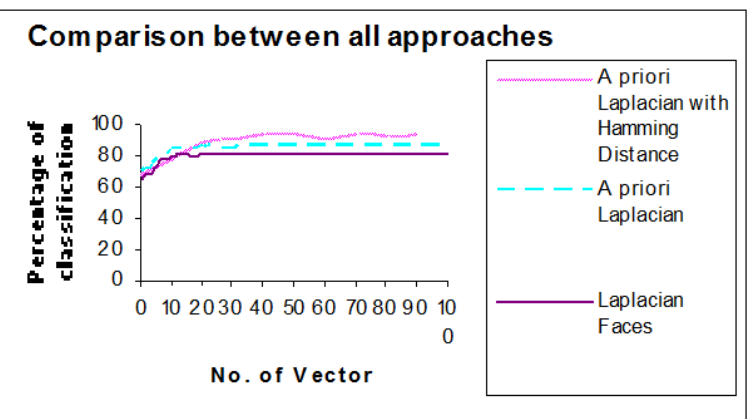

Figure 9 Comparison of three approaches w.r.t. No. of Eigen vectors

\section{REFERENCES}

[1] Gaurav Gupta and Vishal Gupta "A Priori Laplacian Concept: An Enhanced method for face recognition". International Journal of Advanced Research in Computer Science, Volume 2, No.4, July-August 2011.

[2] A.M. Martinez and A.C. Kak, "PCA versus LDA," IEEE Trans. Pattern Analysis and Machine Intelligence, vol. 23, no. 2, pp. 228-233, Feb. 2001

[3] M. Turk and A.P. Pentland, "Face Recognition Using Eigenfaces," IEEE Conf. Computer Vision and Pattern Recognition, 1991.

[4] X. He and P. Niyogi, "Locality Preserving Projections," Proc. Conf. Advances in Neural Information Processing Systems, 2003.

[5] Xiaofei He, Shuicheng Yan, Yuxiao Hu, Partha Niyogi, and Hong-Jiang Zhang, Fellow, IEEE, "Face Recognition Using Laplacianfaces ," IEEE Trans. On Pattern Analysis and Machine Intelligence, vol. 27, no. pp 328340, March 2005.

[6] R. Chellappa, C. L. Wilson and S. Sirohey, "Human and Machine Recognition of Faces: A Survey", Proceedings of the IEEE, vol. 83, no. 5, May 1995.

[7] K. Etemad, and R. Chellappa, "Face Recognition Using Discriminant Eigenvectors”, pp. 2148-2151, IEEE, 1996.

[8] W. Zhao, A. Krishnaswamy, R. Chellappa, D. L. Swets, and J. Weng, "Discriminant Analysis of Principal Components for Face Recognition”, pp. 336-341, International Conference on Automatic Face and Gesture Recognition, 1998.

[9] R. O. Duda, P. E. Hart, and D. G. Stork, "Pattern Classification”, John Wiley \& Sons, 2nd Edition, 2001.

[10] Face Database from AT\&T Laboratories Cambridge http://www.cl.cam.ac.uk/Research/DTG/attarchive:pub/d ata/att_faces.zip.

[11] B. Srinivasa Reddy and B. N. Chatterji, "An FFT-Based Technique for Translation, Rotation, and Scale-Invariant Image Registration", IEEE Trans. on Image Processing, Vol. 5, No. 8, August 1996 pp 1266-1271.

[12] Jiarui Lin, Zhiyong Gao, Bangquan Xu, Yangxiezi Cao, Zhan yingjian, "The Affection of Grey Levels on Mutual Information Based Medical Image Registration", Proceedings of the 26th Annual International Conference of the IEEE EMBS San Francisco, CA, USA September 1-5, 2004 pp 1747-1750. 
[13] A. X. Guan, and H. H. Szu, "A Local Face Statistics Recognition Methodology beyond ICA and/or PCA", pp. 1016-1027, IEEE, 1999.

[14] P. J. Phillips, "Support Vector Machines Applied to Face Recognition”, pp. 803-809, Advances in Neural Information Processing Systems 11, MIT Press, 1999.

[15] C. Podilchuk, and X. Zhang, "Face Recognition Using DCT Based Feature Vectors", pp. 2144-2147, IEEE, 1996.
[16] Shashua, A. Levin, and S. Avidan, "Manifold Pursuit: A New Approach to Appearance Based Recognition," Proc. Int'l Conf. Pattern Recognition, Aug. 2002.

[17] Peter N. Belhumeur, Joao P. Hespanha, and David J. Kriegman, "Eigenfaces vs. Fisherfaces: Recognition Using Class Specific Linear Projection," IEEE Trans. On Pattern Analysis And Machine Intelligence, Vol. 19, No. 7, pp 711-720 July 1997. 